\title{
Exposure to bisphenol A induces dysfunction of insulin secretion and apoptosis through the damage of mitochondria in rat insulinoma (INS-1) cells
}

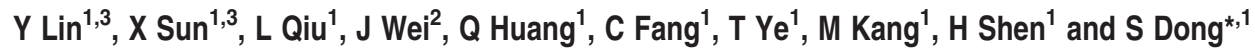

Bisphenol $A$ (BPA) is widely used in plastic products, through which humans are exposed to it. Accumulating evidence suggests that BPA exposure is associated with $\beta$-cell dysfunction. Mitochondrial defects can cause impairment and failure of $\beta$ cells, but there is little information about the effects of BPA on the mitochondrial function of $\beta$ cells. In this study, we assessed the role of mitochondria-mediated mechanisms underlying BPA-induced $\beta$-cell dysfunction and resulting $\beta$-cell apoptosis. INS-1 cells were cultured with $0,0.0020,0.020,0.20$, or $2.0 \mu \mathrm{M}$ BPA. Cell viability, glucose-stimulated insulin secretion (GSIS), and mitochondrial function were examined. The mitochondrial apoptotic pathway was also analyzed at molecular level. We found that BPA suppressed cell viability and disturbed GSIS in a dose-dependent manner. Positive Annexin- propidium iodide (PI) staining and altered expression of Bcl-2 family members and caspases in INS-1 cells indicated that the cells progressively became apoptotic after BPA exposure. Additionally, BPA-induced apoptosis was associated with mitochondrial defects in $\beta$ cells, as evidenced by depletion of ATP, release of cytochrome $c$, loss of mitochondrial mass and membrane potential, and alterations in expression of genes involved in mitochondrial function and metabolism. Taken together, these findings provide strong evidence that BPA triggers INS-1 cells dysfunction and apoptosis may be meditated via the mitochondrial pathway.

Cell Death and Disease (2013) 4, e460; doi:10.1038/cddis.2012.206; published online 17 January 2013

Subject Category: Experimental medicine

Bisphenol A (BPA) is an environmental endocrine disruptor extensively used in the manufacture of food and beverage containers, cigarette filters, thermal (carbonless) receipts, and paper currency. ${ }^{1}$ The human population is continuously and inevitably exposed to BPA from multiple sources, and $92.6 \%$ of the population aged six and older in the United States have detectable levels of BPA in their urine and blood. ${ }^{2,3}$ Recently, the potential effects of BPA exposure on the risk of type 2 diabetes and insulin resistance have been of great interest. ${ }^{4,5}$ Epidemiological studies using data from the 2003-2004 National Health and Nutrition Examination Survey (NHANES) first reported a positive association between increased levels of urinary BPA and diabetes diagnosis. ${ }^{4}$ However, follow-up analysis of the NHANES 2005-2008 report showed an inconsistent association between the prevalence of type 2 diabetes and the concentration of BPA in urine. ${ }^{6,7}$ Interestingly, when all three NHANES studies were combined and analyzed together, urinary BPA levels were significantly associated with diabetes mellitus independent of traditional risk factors. ${ }^{7,8}$ In China, a community-based investigation conducted between 2008 and 2009 indicated an association between the highest urinary levels of BPA and diabetes but no clear association between diabetes and the presence of BPA in the urine at lower levels. ${ }^{9}$ Therefore, more longitudinal and long-term prospective studies are urgently needed to further investigate whether there is a true association between BPA exposure and type 2 diabetes.

Although the role of BPA as a risk factor for diabetes mellitus remains controversial, accumulating studies in animals and in cellular models demonstrate the physiological plausibility of BPA's adverse effect on $\beta$-cell function and the whole body glucose homeostasis. As just one example, administration of $100 \mu \mathrm{g} / \mathrm{kg} /$ day BPA for 8 days in adult mice led to insulin resistance. ${ }^{10}$ Moreover, significantly increased glucose-stimulated insulin secretion (GSIS) and insulin content were detected in pancreatic $\beta$ cells of male mice exposed to $100 \mu \mathrm{g} / \mathrm{kg} /$ day BPA for 4 days, implying that BPA directly affected $\beta$-cell function in addition to affecting peripheral tissue. Indeed, several published studies confirm that pancreatic $\beta$ cells are a target of BPA. For instance, glucose-induced $\mathrm{Ca}^{2+}$ signals were potentiated, and the transcription factor CREB was activated in freshly isolated islets treated with a low dose of BPA. ${ }^{11,12}$ Increased insulin content and release, as well as changes in Insulin gene transcription, had also been demonstrated in an $E R \alpha$ dependent manner in BPA-treated isolated islets. ${ }^{13}$ In a more recent study, this group further highlighted the impact of BPA on $\beta$ cells, indicating that BPA induced a rapid reduction in $\mathrm{K}_{\text {ATP }}$ channel activity and induced insulinotropic effects in mice and human islets through nuclear $\operatorname{ER} \beta .{ }^{14}$ All of these

\footnotetext{
${ }^{1}$ Key Lab of Urban Environment and Health, Department of Environmental and Molecular Toxicology, Institute of Urban Environment, Chinese Academy of Sciences, Xiamen 361021, China and ${ }^{2}$ Ministry of Education Key Laboratory for Cell Biology and Tumor Cell Engineering, Department of Biomedical Sciences, School of Life Sciences, Xiamen University, Xiamen 361005, China

${ }^{*}$ Corresponding author: S Dong, Urban Environment, Chinese Academy of Sciences, Key Lab of Urban Environment and Health, Institute of Urban Environment, Chinese Academy of Sciences, Xiamen, Fujian 361021, China. Tel: + 86 05926190779; Fax: + 86 05926190566; E-mail: sjdong@iue.ac.cn

${ }^{3}$ These authors contributed equally to this work.

Keywords: bisphenol A; INS-1 cells; insulin secretion; mitochondrial dysfunction; apoptosis

Received 21.9.12; revised 25.11.12; accepted 04.12.12; Edited by M Federici
} 
data confirmed that BPA could regulate insulin biosynthesis and secretion in $\beta$ cells via nuclear ER, but other molecular and cellular pathways involved in BPA-induced $\beta$-cell toxicity must be further elucidated.

One of the key organelles in pancreatic $\beta$ cells is the mitochondrion, which plays a central role in the generation of ATP and other factors linking glucose metabolism to insulin secretion. ${ }^{15-17}$ In a previous study, mitochondrial damage was observed in $\beta$ cells at weaning in Wistar rat offspring exposed perinatally to $50 \mu \mathrm{g} / \mathrm{kg} /$ day of BPA. Furthermore, the early mitochondrial defects observed in BPA-exposed offspring worsened with age, causing progressive loss of pancreatic islet function, development of insulin resistance, and pre-diabetes later in life, even though BPA exposure was discontinued at weaning. ${ }^{18}$ In the case of isolated rat islets, BPA exposure also induced remarkable swelling of the mitochondria with loss of distinct crista structure, and these changes were accompanied by decreases in ATP and the mitochondrial respiratory enzyme COX IV. ${ }^{19}$ These findings suggest that BPA-induced mitochondrial defects may play a critical role in the pathology of glucometabolic disorders.

Considering that a large amount of cellular material from $\beta$ cells was factored into biochemical determinations, the present study used a rat insulinoma (INS-1) cell model ${ }^{20}$ to explore the hypothesis that exposure of $\beta$ cells to BPA leads to mitochondrial dysfunction, subsequently attenuates insulin secretion, and ultimately triggers apoptosis.

\section{Results}

BPA decreases cell viability in INS-1 cells. INS-1 cells exposed to 0.0020 or $0.020 \mu \mathrm{M}$ BPA for $12 \mathrm{~h}$ did not exhibit any significant change in cell viability, whereas at higher concentrations $(0.20$ or $2.0 \mu \mathrm{M})$, cell viability was significantly decreased to 85.1 or $55.1 \%$ of that observed in control cells, respectively (Figure 1a). When the incubation time was extended to $24 \mathrm{~h}, 0.020,0.20$, or $2.0 \mu \mathrm{M}$ BPA significantly reduced the viability of INS-1 cells to $83.4,69.3$, or $47.1 \%$ of that observed in unstimulated control cells, respectively. A further decrease in cell viability was observed after $48 \mathrm{~h}$ of BPA incubation at all concentrations.

BPA impairs GSIS and alters genes expression of components of classic GSIS pathway in INS-1 cells. As shown in Figure 1b, insulin secretion in response to glucose in INS-1 cells differed between cells treated with low and high levels of BPA. Incubating cells for $48 \mathrm{~h}$ with $0.0020 \mu \mathrm{M}$ BPA did not significantly affect basal insulin release $(5.6 \mathrm{mM}$ glucose); whereas the secretion response evoked by $16.7 \mathrm{mM}$ glucose was significantly elevated in BPA-exposed cells. Compared with untreated control cells, cells exposed to $0.020 \mu \mathrm{M}$ BPA displayed a $122 \%$ increase in basal insulin release but exhibited a slight inhibition of the secretory response to $16.7 \mathrm{mM}$ glucose. In contrast to the increased GSIS, basal insulin secretion tended to be abrogated in cells exposed to $2.0 \mu \mathrm{M}$ BPA. Cells exposed to either 0.20 or 2.0 $\mu \mathrm{M}$ BPA secreted markedly less insulin when cultured in $16.7 \mathrm{mM}$ glucose than did untreated control cells. Consistent with GSIS, the amount of insulin mRNA was found to be slightly increased in cells exposed to $0.0020 \mu \mathrm{M}$ BPA, whereas it was significantly decreased in the cells exposed to $0.020,0.20$, or $2.0 \mu \mathrm{M}$ BPA when compared with controls (Figure 1c). This effect was further confirmed at the protein level by western blot analysis. Treatment of cells with 0.020 , 0.20 , or $2.0 \mu \mathrm{M}$ BPA induced progressive decreases in the insulin protein level (Figure 1c).

Next, real-time RT-PCR was used to evaluate the mRNA levels of genes involved in the classic GSIS pathway. Figure $1 \mathrm{~d}$ and e show that the amounts of mRNA of the major glucose transporter Glut2 and the metabolic enzyme Gck were not affected by 0.0020 or $0.020 \mu \mathrm{M}$ BPA in INS-1 cells, but they were significantly reduced after exposure to 0.20 or $2.0 \mu \mathrm{M}$ BPA. Treatment with $0.0020 \mu \mathrm{M}$ BPA increased the mRNA levels of Kir6.2 and Sur, but they were significantly decreased when the cells were incubated with $0.020,0.20$, or $2.0 \mu \mathrm{M} \mathrm{BPA}$ (Figure 1f and $\mathrm{g}$ ).

BPA induces mitochondrial dysfunction in INS-1 cells. Because the mitochondrion is one of the most important organelles for $\beta$-cell function and survival, its morphology, mass, membrane potential $(\Delta \psi \mathrm{m})$, and ATP generation were measured to evaluate the effects of BPA on mitochondrial function. As shown in Figure $2 \mathrm{a}$, mitochondria were abundant and appeared interconnected in a network throughout the cytoplasm in untreated control cells. That network gradually became fragmented and less organized in cells exposed to BPA, as characterized by dispersed staining. As the signal from MitoTracker Green (MTG) also reflected changes in mitochondrial mass, its fluorescence density was measured. Figure $2 b$ revealed that the average density of MTG in BPAexposed cells was decreased in a dose-dependent manner compared with that measured in controls. Consistent with these findings, cellular ATP levels were reduced to 49.0, 40.3 or $17.3 \%$ of that detected in untreated control cells after incubation $0.020,0.20$, or $2.0 \mu \mathrm{M}$ BPA, respectively. No difference in ATP production was observed between cells treated with $0.0020 \mu \mathrm{M}$ BPA and unexposed control cells (Figure 2c). Further, the fluorescent mitochondrial probe $\mathrm{JC}-1$ was used to measure mitochondrial $\Delta \psi \mathrm{m}$, which is essential for ATP formation and is a marker of mitochondrial membrane integrity. Apoptotic or unhealthy cells with collapsed mitochondria contain mainly green JC-1 monomers rather than red JC-1 clusters. After cells were treated with 0.20 or $2.0 \mu \mathrm{M}$ BPA, a shift of fluorescence from red to green was observed (Figure 2d), indicating that mitochondrial depolarization was induced by BPA $(0.20$ and $2.0 \mu \mathrm{M})$ in INS-1 cells.

The expression of genes involved in mitochondrial function and metabolism was also evaluated. Figure 3 showed that treatment of INS-1 cells with 0.20 or $2.0 \mu \mathrm{M}$ BPA for $48 \mathrm{~h}$ decreased the expression of Tfam, a gene providing a molecular basis for the connection between environmental stimuli and mitochondrial biogenesis and respiration. No difference was detected in the expression levels of the mtDNA-encoded gene $\mathrm{Nd} 4 \mathrm{l}$ between BPA-exposed and control cells (Figure 3). The expression of Atp6, a subunit of the mitochondrial pump ATP synthase, was significantly downregulated in cells exposed to $0.020,0.20$, or $2.0 \mu \mathrm{M}$ BPA when compared with untreated control cells (Figure 3 ). 


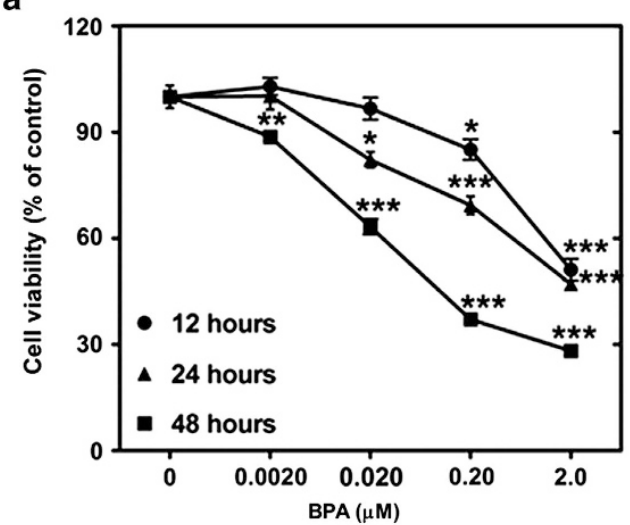

b

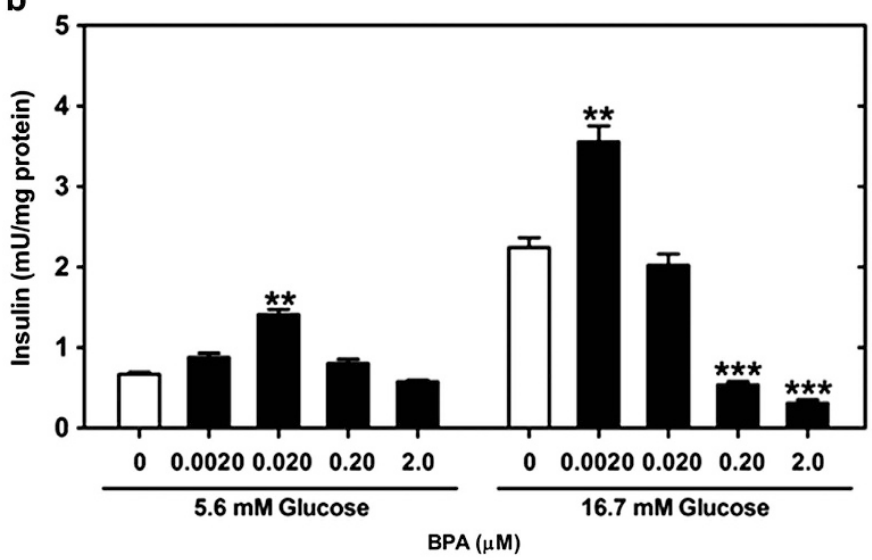

C


Figure 1 BPA decreases cell viability and disrupts GSIS. (a) Cell viability as determined by MTT assay. INS-1 cells were cultured in the presence or absence of BPA $(0.0020-2.0 \mu \mathrm{M})$ for 12,24 , or $48 \mathrm{~h}$. The data are expressed as percentages of untreated control cells. (b) Insulin secretion triggered by glucose after $48 \mathrm{~h}$ BPA exposure. The levels of secreted insulin were normalized to protein content. (c) Relative mRNA and protein expression of insulin in INS-1 cells exposed to BPA for $48 \mathrm{~h}$. (d-g) Expression of genes involved in the GSIS pathway. The results are expressed as the mean \pm S.E.M. of $3-6$ replicates from three independent experiments. ${ }^{\star} P<0.05$; ${ }^{\star} P<0.01$;

${ }^{* \star *} P<0.001$ compared with untreated control cells

Citrate synthase, one of the genes involved in the TCA cycle, was also dramatically suppressed in INS-1 cells after exposure to $0.020,0.20$, or $2.0 \mu \mathrm{M}$ BPA (Figure 3 ). Additionally, the expression of Ucp2 was increased, and the expression of Ogdh was suppressed in INS-1 cells in a dose-dependent manner after $48 \mathrm{~h}$ BPA treatment (Figure 3).

BPA at higher concentrations triggers apoptosis and the release of apoptogenic factors in INS-1 cells. To examine whether BPA caused cell apoptosis, INS-1 cells were stained with Annexin V-PI and analyzed by flow cytometry. Figure 4 showed that exposure to BPA for $48 \mathrm{~h}$ resulted in a dosedependent increase in the proportion of early apoptotic and apoptotic cells compared with controls. Moreover, the mitochondrial and cytosolic fractions were prepared from
INS-1 cells to investigate whether the release of cytochrome $c$ was involved in the apoptotic process induced by BPA. As shown in Figure 5, protein expression of cytochrome $c$ was reduced with increasing BPA concentrations in isolated mitochondria. Likewise, cytochrome $c$ was significantly elevated in the cytosolic fractions in INS-1 cells exposed to $0.020,0.20$, or $2.0 \mu \mathrm{M}$ BPA for $48 \mathrm{~h}$. BPA promoted the translocation of cytochrome $c$ from the mitochondria into the cytosol in INS-1 cells.

BPA triggers apoptosis through the intrinsic mitochondrial pathway. To further determine whether the mitochondrial pathway mediated BPA-induced apoptosis, the expressions of Bcl-2 family members, Apaf-1, and caspases were evaluated. As shown in Figure 6a, BPA significantly 




0.0020

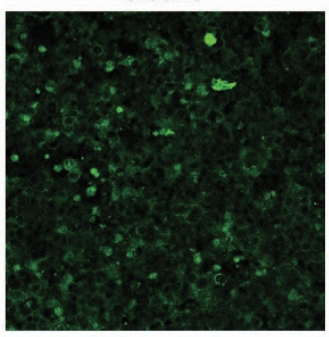

0.020

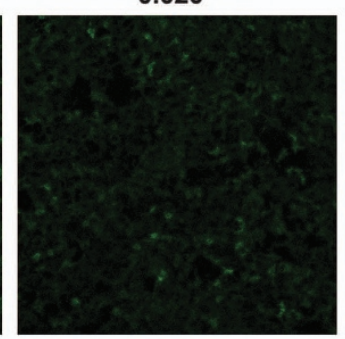

0.20



2.0

$\operatorname{BPA}(\mu \mathrm{M})$
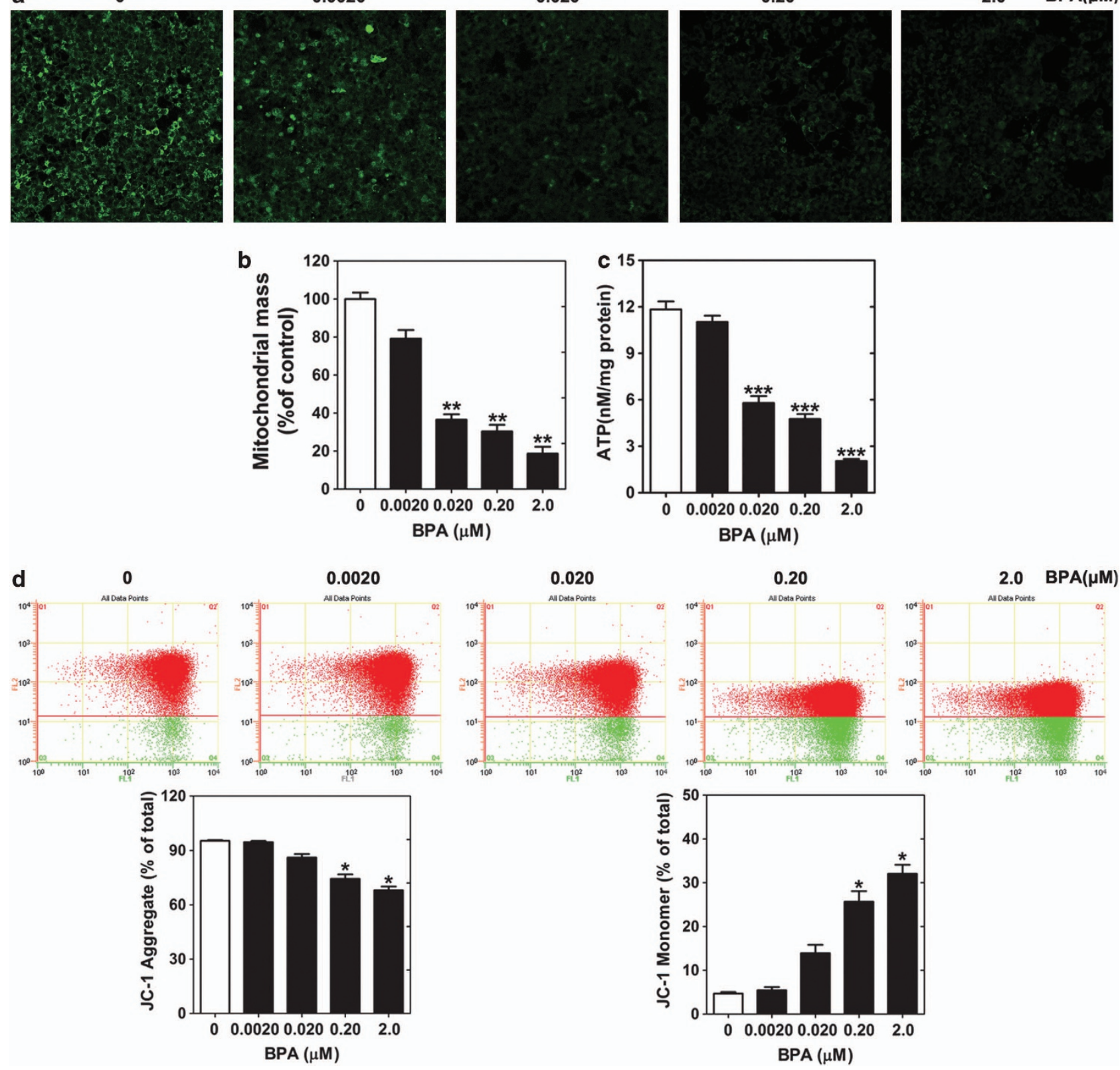

Figure 2 BPA triggers mitochondrial dysfunction. INS-1 cells were cultured in the presence or absence of BPA (0.0020-2.0 $\mu \mathrm{M})$ for $48 \mathrm{~h}$. (a) Representative images of mitochondria in cells stained with MitoTracker Green at $200 \times$ magnification. (b) Quantitative results of (a). The data are expressed as a percentage of the MitoTracker Green staining in untreated control cells. (c) Intracellular ATP content normalized to protein content. (d) Mitochondrial $\Delta \psi \mathrm{m}$ measured by the JC-1 probe. The dot-plot representation of the flow cytometry analysis shows the distribution of JC-1 aggregates (cells emitting red fluorescence in the FLI-2 channel) and JC-1 monomers (cells emitting green JC-1 detected in the FLI-1 channel). The bar graphs show the percentage of JC-1 aggregate-positive and JC-1 monomer-positive cells. The results are expressed as the mean \pm S.E.M. of $2-3$ replicates from three independent experiments. ${ }^{*} P<0.05 ;{ }^{* *} P<0.01 ;{ }^{* * \star} P<0.001$ compared with untreated control cells

increased the expression of the pro-apoptotic protein Bax and moderately decreased the expression of the antiapoptotic protein $\mathrm{Bcl}-2$ in dose-dependent manners. Additionally, markedly increased levels of Apaf-1 and the $17-\mathrm{kDa}$ cleaved form of caspase- 9 were observed in cells treated with $0.020,0.20$, or $2.0 \mu \mathrm{M}$ BPA. There was no change in the expression of the $40-\mathrm{kDa}$ form of cleaved caspase-9 (Figure $6 \mathrm{~b}$ ). Both the $17-\mathrm{kDa}$ and the $19-\mathrm{kDa}$ form of cleaved caspase-3 displayed marked dose-dependent increases in BPA-exposed cells compared with controls (Figure 6b).

\section{Discussion}

BPA is a ubiquitous environmental pollutant that is reported to be associated with an increased incidence of diabetes mellitus and increased damage to $\beta$-cell function. In this study, we 



Figure 3 BPA alters the mRNA expression of genes involved in mitochondrial function and metabolism. INS-1 cells were cultured in the presence or absence of BPA $(0.0020-2.0 \mu \mathrm{M})$ for $48 \mathrm{~h}$. The results are expressed as the mean \pm S.E.M. of duplicates from three independent experiments. ${ }^{*} P<0.05 ;{ }^{* *} P<0.01 ;{ }^{* * *} P<0.001$ compared with untreated control cells
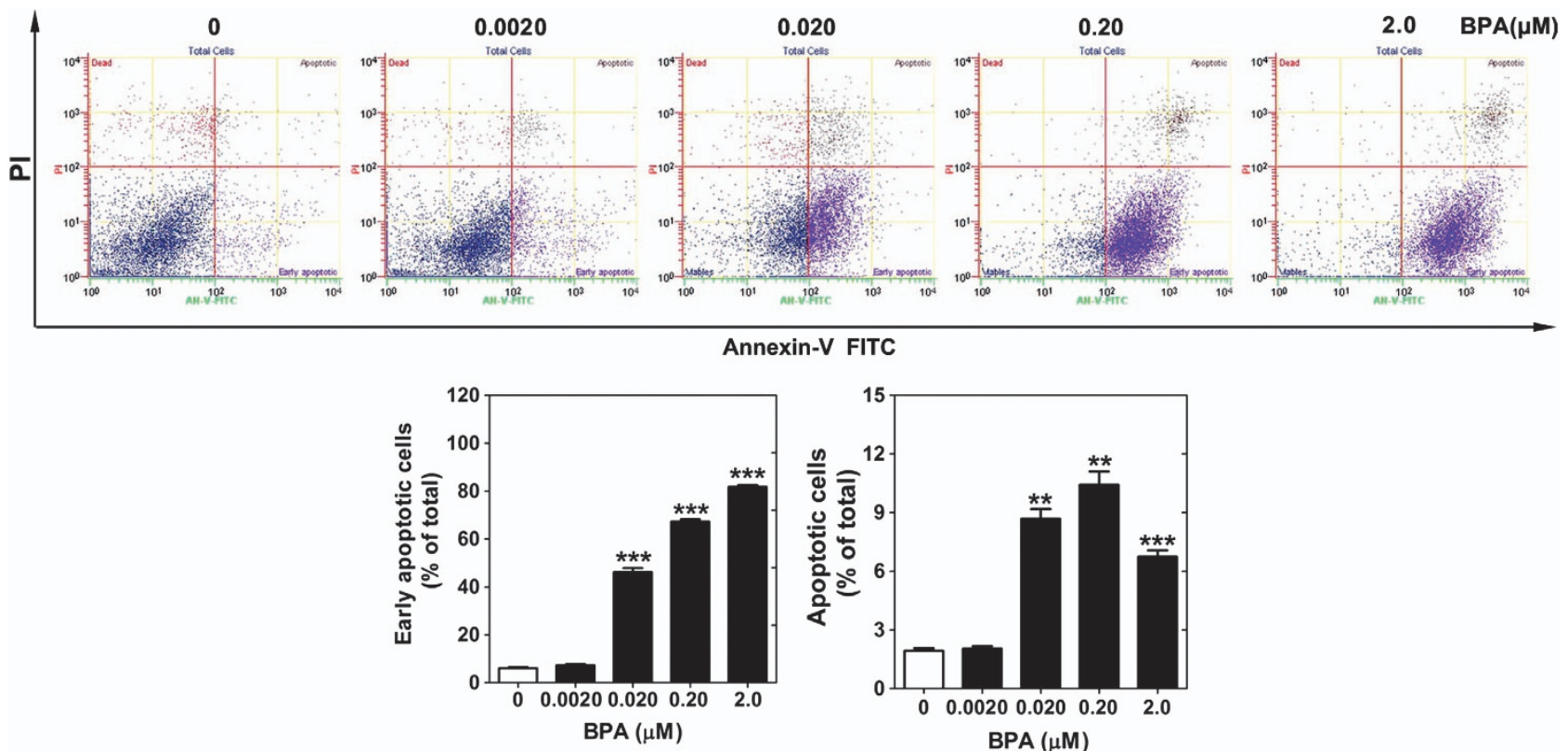

Figure 4 BPA induces dose-dependent apoptosis. INS-1 cells were cultured in the presence or absence of BPA $(0.0020-2.0 \mu \mathrm{M})$ for $48 \mathrm{~h}$. The cells in the bottom right quadrant $(\mathrm{FITC}+$ and $\mathrm{PI}-$ ) and the upper right quadrant $(\mathrm{FITC}+$ and $\mathrm{PI}+$ ) represent early apoptotic and apoptotic cells, respectively. All data are presented as the mean \pm S.E.M. of three independent experiments. ${ }^{\star \star} P<0.01 ;{ }^{* \star \star} P<0.001$ compared with untreated control cells

reported that exposure to BPA $(0.020-2.0 \mu \mathrm{M})$ decreased rat insulinoma (INS-1) cells' viability, disrupted cell function, and, furthermore, increased cell apoptosis in a dose-dependent manner. More importantly, we found that BPA triggered apoptotic signaling in INS-1 cells via a mitochondria-mediated pathway (Figure 7), as shown by mitochondrial fragmentation, the reduction of mitochondrial mass and $\Delta \psi$, the depletion of
ATP, the release of cytochrome $c$ from the mitochondria into the cytosol, and the activation of caspases. Despite the fact that the concentration required for these effects was higher than environmentally relevant levels $(1 \mathrm{nM})$ and the mean serum or urine concentrations reported in bio-monitoring studies, it was still within the reported ranges for some populations, such as factory workers exposed to higher levels 

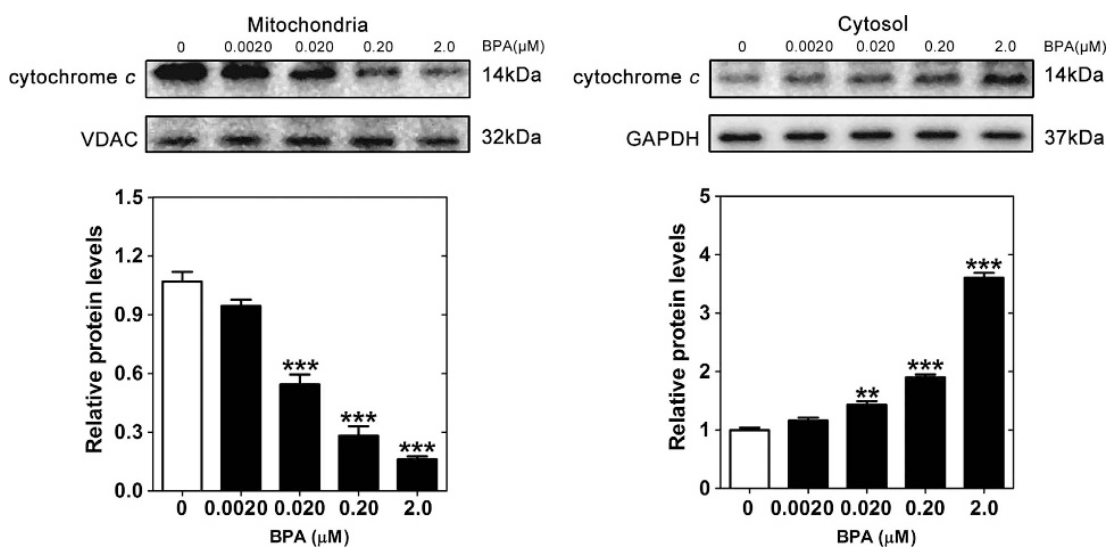

Figure 5 BPA induces the release of cytochrome $c$ from the mitochondria to the cytosol. INS-1 cells were cultured in the presence or absence of BPA (0.0020-2.0 $\mu$ M) for $48 \mathrm{~h}$. (a) Representative western blot for protein expression of cytochrome $c$ in mitochondria. VDAC served as a loading control. (b) Representative western blot for protein expression of cytochrome $c$ in the cytosol. GAPDH served as a loading control. All data are presented as the mean \pm S.E.M. of duplicates from three independent experiments. ${ }^{* \star} P<0.01 ;{ }^{* \star *} P<0.001$ compared with untreated control cells

a



b

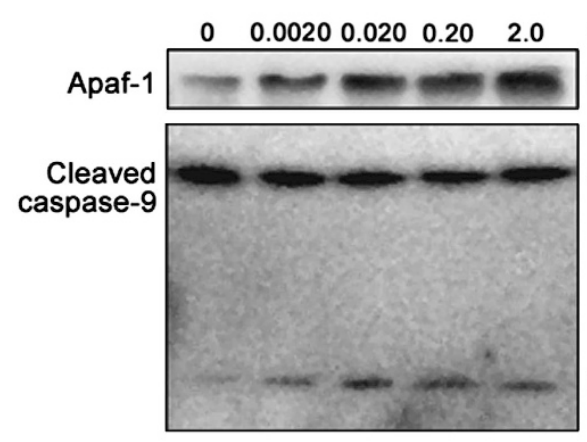

$\mathrm{BPA}(\mu \mathrm{M})$

$135 \mathrm{kDa}$

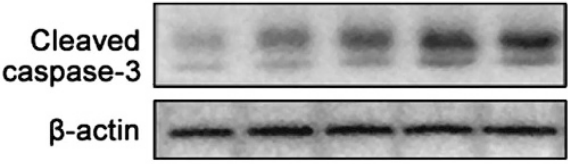

$17 \mathrm{kDa}$
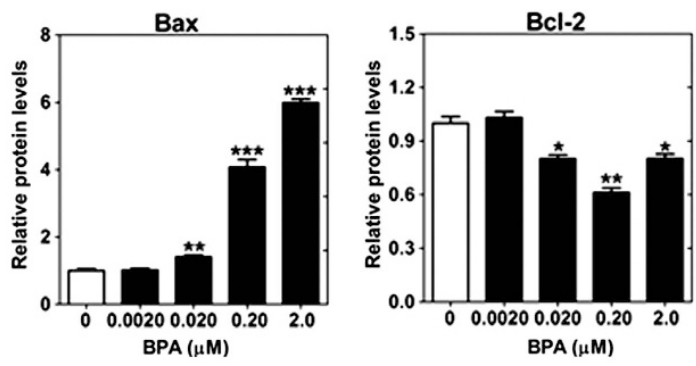
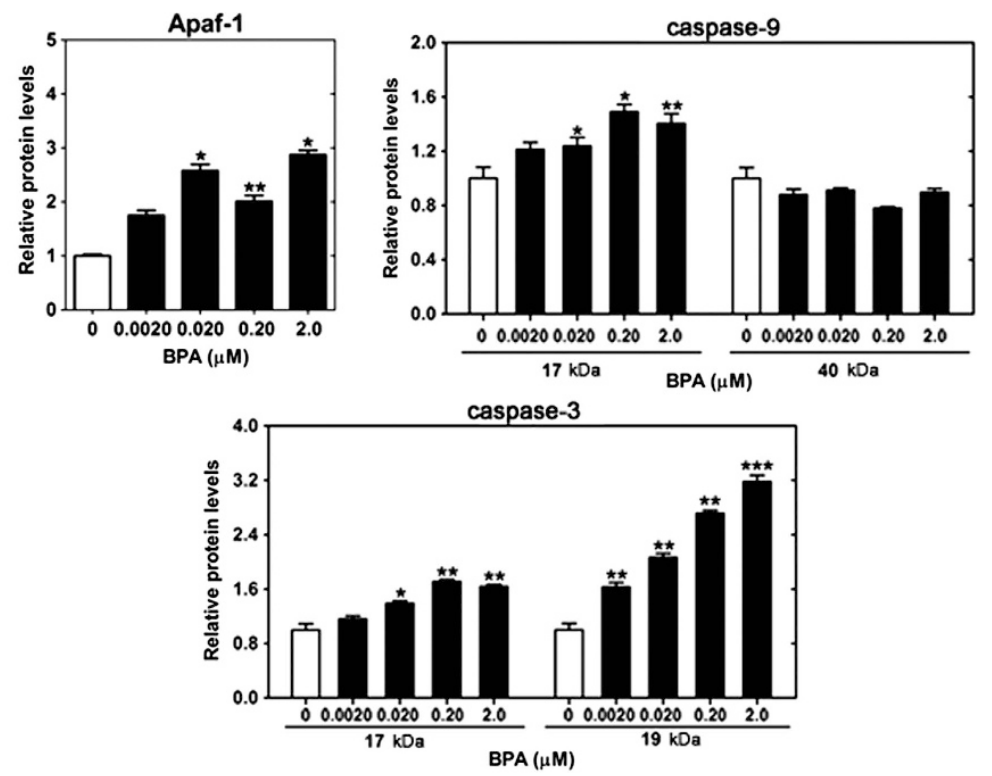

Figure 6 BPA triggers apoptosis through a caspase-dependent mitochondrial pathway. INS-1 cells were cultured in the presence or absence of BPA $(0.0020-2.0 \mu \mathrm{M})$ for $48 \mathrm{~h}$. (a) Western blot analysis of Bax and Bcl-2 expression in cell lysates. (b) Western blot analysis of Apaf-1, cleaved caspase-9, and cleaved caspase-3 in cell lysates. All protein expression levels were normalized relative to a $\beta$-actin loading control. The data are presented as the mean \pm S.E.M. of duplicates from three independent experiments. ${ }^{*} P<0.05 ;{ }^{* *} P<0.01 ;{ }^{* * *} P<0.001$ compared with untreated control cells

of BPA. ${ }^{21-23}$ Moreover, the dose chosen in this study can be considered a 'low dose' because estimates of circulating levels of BPA at the LOAEL define an equivalent low-dose concentration as $<2.19 \times 10^{-7} \mathrm{M}$ in vitro culture studies. ${ }^{24,25}$
In this study, the effects of BPA on cell viability were first determined by the MTT assay, which showed that BPA significantly reduced INS-1 cell viability in a dose- and timedependent manner. We next examined GSIS and found that 


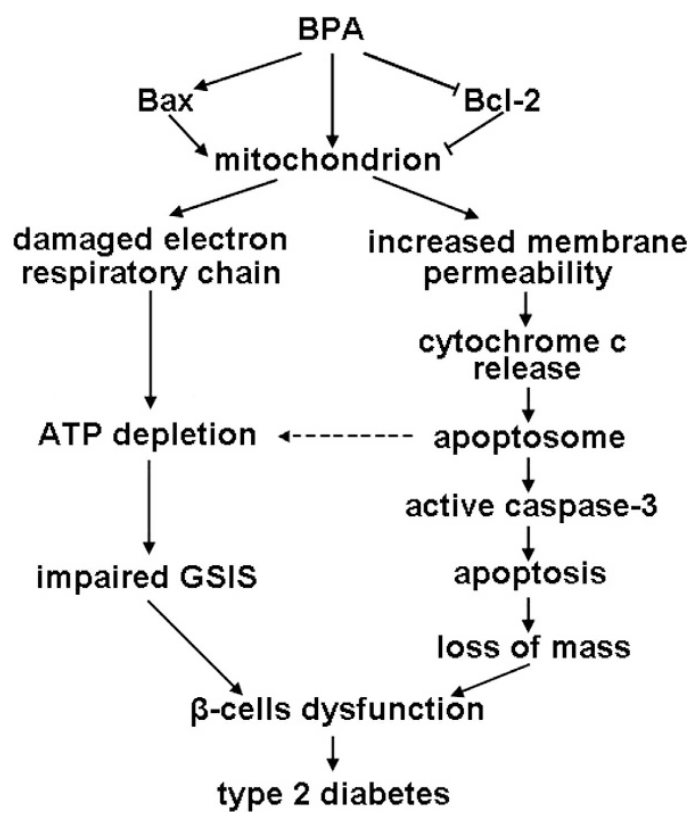

Figure 7 A proposed signaling pathway for BPA-induced $\beta$-cell dysfunction and apoptosis. Mitochondrial damage is triggered by BPA that either directly damages the mitochondria or alters the levels of pro- and anti-apoptotic Bcl-2 family proteins. On the one hand, BPA damages the mitochondrial electron respiratory chain complexes, resulting in the depletion of ATP and the impairment of GSIS in $\beta$ cells. On the other hand, damaged mitochondria release cytochrome $c$ to the cytosol. Cytochrome $c$ consequently triggers the formation of apoptosomes, the activation of caspases, and finally, the execution of apoptosis in $\beta$ cells. Arrows in the figure represent stimulative effects, and $\mathrm{T}$ lines represent inhibitory effects

cells exposed to 0.20 or $2.0 \mu \mathrm{M}$ BPA exhibited a significantly diminished ability to secrete insulin in response to glucose stimulation, even though insulin secretion was slightly increased after exposure to 0.0020 or $0.020 \mu \mathrm{M}$ BPA. The reactive insulin stimulatory effects induced by exposure to lower doses of BPA could be considered cellular adaptive responses to BPA stimulus. However, INS-1 cells were unable to compensate for higher levels of BPA, resulting in significant declines in GSIS as well as decreased mRNA and protein expression of insulin. These observations are in agreement with a prior report that environmentally relevant doses of BPA (1 nM) could stimulate GSIS in human and mouse islets ${ }^{14}$ and are highly consistent with a recent study showing that short-term exposure ( $24 \mathrm{~h}$ ) to 0.11 or $1.1 \mu \mathrm{M} \mathrm{BPA}$ reduced insulin secretion and insulin content in isolated rat islets. ${ }^{19} \mathrm{~A}$ nonlinear dose-response relationship may exist between BPA and the impairment of GSIS, but this remains to be elucidated in our future research.

In $\beta$ cells, glucose is transported by Glut2, phosphorylated by Gck, and converted to pyruvate by glycolysis. In the mitochondria, pyruvate enters the TCA cycle and activates ATP formation, which promotes the closure of ATP-sensitive $\mathrm{K}$ ( $\left.\mathrm{K}_{\text {ATP }}\right)$ channels and the depolarization of the plasma membrane and stimulates insulin secretion. ${ }^{26,27}$ Impaired GSIS can be mediated by reduced glucose sensing, disrupted mitochondrial metabolism, or a combination of both. In this study, we did not find evidence that exposure to lower concentrations of BPA $(0.0020$ or $0.020 \mu \mathrm{M})$ altered the expression of Glut2 and Gck in INS-1 cells, despite the fact that treatment with 0.20 or $2.0 \mu \mathrm{M}$ BPA downregulated both genes at the mRNA level. Thus, it was not clear whether BPA impaired GSIS mainly through regulating the rate of glucose uptake and glycolysis. The downregulation of Gck and Glut2 in INS-1 cells treated with the highest concentrations $(0.20$ or $2.0 \mu \mathrm{M}$ ) of BPA was most likely a consequence rather than a cause of the impaired GSIS observed in BPA-exposed cells. On the other hand, treatment with $0.0020 \mu \mathrm{M}$ BPA moderately increased the expression of the $\mathrm{K}_{\text {ATP }}$ channel subunits Kir6.2 and Sur, which may account, at least in part, for the elevated insulin secretion observed in INS-1 cells exposed to BPA at this concentration. Conversely, the expression levels of these two genes were suppressed in INS-1 cells after incubation with as little as $0.020 \mu \mathrm{M}$ BPA, and this effect was accompanied by a significant depletion of ATP. Because it has been estimated that $98 \%$ of the ATP in $\beta$ cells is produced by mitochondrial oxidative metabolism, ${ }^{28}$ we hypothesized that BPA-induced mitochondrial damage might play a critical role in the development of $\beta$-cell dysfunction.

Indeed, a recent study indicated that Wistar rat offspring perinatally exposed to $50 \mu \mathrm{g} / \mathrm{kg} /$ day BPA present profound mitochondrial structural defects in $\beta$ cells that precede any observable alterations in glucose homeostasis. ${ }^{18}$ Moreover, exposure of isolated rat islets to $0.11 \mu \mathrm{M}$ BPA has also been reported to cause mitochondrial swelling, to alter the expression of typical mitochondrial genes, to decrease the activity of the mitochondrial respiratory enzyme COX IV, and to reduce ATP content. ${ }^{19}$ In line with these results, we found that several key events involved in the regulation of mitochondrial function and metabolism were damaged by BPA in INS-1 cells. MTG fluorescence showed that BPA destroyed mitochondrial structural integrity and decreased mitochondrial mass in a dose-dependent manner in INS-1 cells. These profound structural defects were accompanied by changes in the regulation of genes that are central to mitochondrial function and energetic metabolism. For example, the expression of Atp6 (subunit 6 of the mitochondrial ATP synthase) and Citrate synthase (one of the genes coding for enzymes involved in rate-limiting steps of the TCA cycle) was significantly decreased in cells exposed to $0.020-2.0 \mu \mathrm{M}$ BPA, partly explaining the decrease in the capacity of the mitochondria to generate ATP after BPA treatment. Meanwhile, downregulation of $O g d h$ and upregulation of $U c p 2$ were observed in BPA-exposed cells. Ogdh encodes a rate-limiting enzyme in the mitochondrial TCA cycle, and Ucp2 has been reported to negatively regulate the sensitivity of insulin secretion to glucose stimulation and the metabolic efficiency of mitochondrial ATP production in $\beta$ cells. ${ }^{29-31}$ Observed changes in the expression of these genes are therefore likely to contribute to the BPA-induced defects in ATP production and GSIS. Tfam encodes a mitochondrial transcription factor that provides a molecular basis for the connection between environmental stimuli and mitochondrial biogenesis. ${ }^{32}$ The reduction in the expression of Tfam observed in BPA-exposed cells thus further underlined a negative effect of BPA on mitochondrial function.

One potential consequence of mitochondrial dysfunction is the increase in apoptosis. It has been well documented that BPA can trigger apoptosis in various cells and tissues through 
mitochondrial signaling pathways. ${ }^{24,33}$ For instance, pubertal BPA exposure resulted in germ cell apoptosis in the testes through not only the Fas/FasL signaling pathway but also the mitochondrial apoptotic pathway. ${ }^{34} \mathrm{~A}$ low dose of BPA was also reported to induce apoptosis in cultured ovarian granulosa cells through the regulation of Bcl-2 family proteins. ${ }^{35}$ In the present study, we found that BPA exposure caused apoptosis in INS-1 cells via activation of the mitochondrial apoptotic pathway, that is, the $\mathrm{Bcl}-2$ family and caspases. The Bcl-2 family, including pro-apoptotic and anti-apoptotic members, has been reported to be a central regulator in the mitochondria-dependent apoptotic pathway. Bax is a typical pro-apoptotic protein that can induce mitochondrial membrane permeability and promote cell death by releasing apoptogenic factors from the mitochondria. In contrast, $\mathrm{Bcl}-2$ is an anti-apoptotic protein that can maintain the integrity of the mitochondrial outer membrane. ${ }^{36,37}$ In the current study, we found that BPA induced the upregulation of Bax expression and the downregulation of $\mathrm{Bcl}-2$ expression, leading to altered mitochondrial membrane permeability, reduced $\Delta \psi$, the release of mitochondrial cytochrome $c$ to the cytosol and the subsequent activation of caspases (Figure 7). The caspase family is a class of cysteine proteases. After being processed into their active forms in cells undergoing apoptosis, caspases play a crucial role in the transduction of apoptotic signals. ${ }^{38}$ Relevant caspases include the initiator caspases (caspases-8, -9 , and -10) and the effector caspases (caspases-3, -6, and -7). When INS-1 cells received BPA stimulus, the cells were responsive to Apaf- 1 activation in a dose-dependent fashion. Apaf-1 interacts with cytochrome $c$ in the cytoplasm to form an apoptosome complex, which in turn activates the caspases that are responsible for the execution of cell death. ${ }^{39}$ Consistent with this hypothesis, BPA triggered the activation of pro-caspase-9, as evidenced by a significant increase in the expression of cleaved (active) caspase-9 in INS-1 cells. Activated caspase-9 remained with the apoptosome as a holoenzyme to maintain its catalytic activity and activated caspase-3, one of the effector caspases involved in the execution of the majority of cellular apoptotic events. ${ }^{39,40}$ All of these findings indicated that the mitochondria-mediated apoptotic signaling pathway was involved in the apoptotic process triggered by BPA in INS-1 cells.

In summary, data from the current study overwhelmingly indicate that BPA affects the viability and function of normal $\beta$ cells and accelerates the rate of apoptosis in INS-1 cells in a dose-dependent manner. Importantly, we suggest that mitochondrial defects and mitochondria-dependent apoptotic signals play vital roles in BPA-induced apoptosis in INS-1 cells (Figure 7). BPA is an important environmental risk factor for diabetes, and precisely defining the mechanisms involved in underlying the toxic action of BPA in $\beta$ cells requires further investigation.

\footnotetext{
Materials and methods

Cell culture and treatments. The INS-1 cells derived from rat insulinoma were purchased from Biohermes (Shanghai, China) and were grown in RPMI 1640 medium supplemented with $10 \%$ FBS, $5.6 \mathrm{mM}$ glucose, $25 \mathrm{mM}$ HEPES, $2 \mathrm{mM}$ L-glutamine, $50 \mu \mathrm{M} \beta$-mercaptoethanol, $100 \mathrm{U} / \mathrm{ml}$ penicillin, and $100 \mu \mathrm{g} / \mathrm{ml}$
}

streptomycin at $37^{\circ} \mathrm{C}$ in a humidified atmosphere containing $95 \%$ air and $5 \%$ $\mathrm{CO}_{2}$. INS-1 cells were used because they are highly responsive to changes in the glucose concentration and have been widely used to evaluate $\beta$-cell function in vitro. ${ }^{20}$ Culture medium, FBS, and supplements were purchased from Invitrogen (Carlsbad, CA, USA).

For experiments, INS-1 cells were seeded at a density of $6.0 \times 10^{3} /$ well in 96-well plates, $2.5 \times 10^{5} /$ well in 24 -well plates, or $6.0 \times 10^{5}$ cells/well in six-well plates. After $24 \mathrm{~h}$, the medium was removed, and the cells were incubated with fresh medium containing serial dilutions of BPA $(0,0.0020,0.020,0.20$, or $2.0 \mu \mathrm{M})$ for a designated period of time. BPA was dissolved in dimethyl sulfoxide (DMSO) because of its poor water solubility. The final concentration of DMSO in medium was $0.1 \%, v / v$.

MTT viability assay. INS-1 cells were seeded in 96-well plates and stimulated with BPA. At the end of the treatment, the medium was removed, and MTT $(0.5 \mathrm{mg} / \mathrm{ml})$ was added to each well for at least $4 \mathrm{~h}$. The purple formazan precipitate was dissolved with DMSO, and the color intensity was measured at $550 \mathrm{~nm}$ with a SpectraMAX M5 microplate reader (Molecular Devices, Sunnyvale, CA, USA).

Glucose-stimulated insulin secretion. INS-1 cells were seeded in 24well plates and exposed to BPA for $48 \mathrm{~h}$. Insulin secretion in response to glucose was assessed as described previously. ${ }^{18}$ Briefly, the treated cells were first preincubated with Krebs-Ringer bicarbonate HEPES buffer (KRBH: $115 \mathrm{mM} \mathrm{NaCl}$,

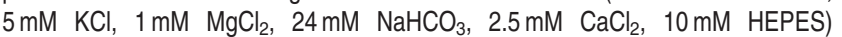
supplemented with $0.5 \%$ BSA and a low level of glucose $(3.0 \mathrm{mM})$ for $1 \mathrm{~h}$. Afterward, the cells were incubated in KRBH buffer containing 5.6 or $16.7 \mathrm{mM}$ glucose for $1 \mathrm{~h}$. The supernatants were collected for the determination of insulin secretion. Insulin concentrations were measured using a Rat Insulin ELISA kit (Millipore, Billerica, MA, USA). The levels of secreted insulin were normalized to the protein content, which was determined with the BCA Protein Assay Kit (Thermo Scientific, Rockford, IL, USA).

ATP assay. INS-1 cells were seeded in six-well plates and incubated with BPA for $48 \mathrm{~h}$ as described above. At the end of the treatment, the cells were collected, and cellular ATP concentrations were determined using an ATP luminometric assay kit (Beyotime Inst. Biotech, Peking, China) following the manufacturer's instructions. Briefly, the cells were mixed with luciferase, which were centrifuged at $10000 \times g$ for $2 \mathrm{~min}$ at $4^{\circ} \mathrm{C}$. The resulting supernatant was then mixed with luciferase reagent, which catalyzed the light production from ATP and luciferin. Luminance (RLU) was linearly related to the ATP concentration and was measured using a SpectraMAX M5 microplate reader. ATP levels were expressed as $\mathrm{nmol} / \mathrm{mg}$ protein.

Measurements of mitochondrial morphology and mass. After treatment, the cells were mixed with $100 \mathrm{nM}$ MTG fluorescent probe (MTG, Beyotime Inst. Biotech) for $30 \mathrm{~min}$ at $37^{\circ} \mathrm{C}$. The probe can be taken up into the mitochondria and will then produce a fluorescence signal. Fluorescence images of cells were obtained with an LSM 710 Laser Scanning Confocal Microscope (Zeiss, Jena, Germany) using a 490-nm laser for excitation and a 510-nm filter for emission. The fluorescence intensity of cells was analyzed with a SpectraMAX M5 microplate reader.

Determination of mitochondrial membrane potential $(\Delta \psi \mathrm{m})$. The

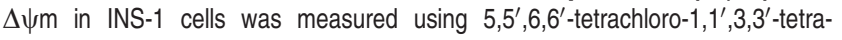
ethylimidacarbocyanine iodide fluorescent probe (JC-1) (Cayman Chemical, Ann Arbor, MI, USA). The treated cells in six-well plates were harvested and incubated with JC-1 at $37^{\circ} \mathrm{C}$ for $15-20 \mathrm{~min}$. Next, the staining solution was removed. The cells were washed and resuspended in phosphate-buffered saline (PBS) and then analyzed with a flow cytometer (Quanta SC, Beckman Coulter, Brea, CA, USA). The loss of $\Delta \psi \mathrm{m}$ was reflected by the increased green fluorescence from JC-1 monomers as well as the loss of red fluorescence from JC-1 aggregates.

Apoptosis assay. An Annexin V-fluorescein isothiocyanate (FITC) staining assay was performed to measure apoptosis in INS-1 cells. The cells were seeded in six-well plates and exposed to BPA for $48 \mathrm{~h}$. The cells were then collected by trypsinization, washed with PBS, and stained with Annexin V-FITC and PI (BioVision, Mipitas, CA, USA) for $15 \mathrm{~min}$ at room temperature. Positive cells were detected with flow cytometry. 
Table 1 Primer sequences for quantiative real-time PCR

\begin{tabular}{|c|c|c|c|c|}
\hline Gene name & Size (bp) & $5^{\prime}$-Primer & $3^{\prime}$-Primer & Accession number \\
\hline Insulin & 166 & CTACAGTCGGAAACCATCAGCA & CCACCAAGTGAGAACCACAAAG & NM_019130.2 \\
\hline Glut2 & 151 & CAGCTGTCTCTGTGCTGCTTGT & GCCGTCATGCTCACATAACTCA & NM_012879.2 \\
\hline Gck & 72 & CACCCCAGAAGGCTCAGAAG & AGCATCACTCTGAAGTTGGTTCCT & NM_012565.1 \\
\hline Kir6.2 & 142 & TGTCCCGAAAGGGCATTATC & ATGTTCTTGTGGGCGACGTT & NM_031358.3 \\
\hline Sur & 142 & CAAGGTCGTAAACCGCAAGC & AGGGGTCCAGGTGAAGAAGC & NM_013039.2 \\
\hline Tfam & 118 & GAAGAGCAAATGGCTGAAGTT & GTGCCCAATCCCAATGAC & NM_031326.1 \\
\hline Nd4l & 103 & TCCCAATTACCATTCTAGTTTT & AGGTTTTGTACGTAGTCTGTTCCGT & EU104720 \\
\hline Atp6 & 164 & GCGTCTGGAGGACCTGTTGA & CCTCAGGACTGGGGTTTGT & BC059121 \\
\hline Citrate synthase & 103 & GCCCTCAACAGTGAAAGCA & TGGCAATCAGGTCCATACAG & NM_130755 \\
\hline Ucp2 & 143 & TGTGGTAAAGGTCCGCTTCC & TTCGGGCAACATTGGGAG & NM_019354.2 \\
\hline Ogdh & 138 & CCGTGCCCGCTGACATTAT & TCTCCCGAAGAGGAAGTGC & NM_001017461.1 \\
\hline $36 B 4$ & 93 & CAGCAGGTGTTTGACAATGGC & TGAGGCAACAGTCGGGTAGC & NM_022402.1 \\
\hline
\end{tabular}

Abbreviations: Glut2, glucose transporter member 2; Gck, glucokinase; Kir6.2, potassium inwardly rectifying channel, subfamily J, member 11; Sur, ATP-binding cassette, subfamily C (CFTR/MRP), member 8; Tfam, transcription factor A, mitochondrial; Nd4l, NADH-ubiquinone oxireductase subunit 4 L; Atp6, ATP synthase subunit 6; Ucp2, Uncoupling protein 2; Ogdh, oxoglutarate ( $\alpha$-ketoglutarate) dehydrogenase (lipoamide).

Total RNA preparation and real-time PCR. INS-1 cells were cultured and treated as described above. Total RNA was extracted using the High Pure RNA kit (Roche, Mannheim, Germany) and was reverse transcribed using PrimeScript TM RT-PCR Kit (Takara, Dalian, China) according to the manual. Real-time PCR was carried out using SYBR Premix Ex Taq II kit (Takara) following the manufacturer's instructions on a LightCycler 480 Instrument (Roche Applied Science). All quantifications were performed with $36 \mathrm{~B} 4$ as an internal standard, and the relative amount of mRNA was calculated using the $2^{-\Delta \Delta C T}$ method. The primer sequences are shown in Table 1.

Western blot analysis. After treatment, INS-1 cells were quickly harvested, rinsed thoroughly with PBS, and then lysed in ice-cold RIPA lysis buffer $(50 \mathrm{mM}$

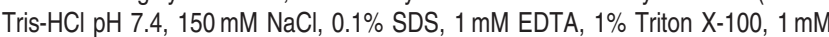
phenylmethylsulfonyl fluoride) for 30-60 min. The lysates were collected and centrifuged at 13000 r.p.m. for $10 \mathrm{~min}$ at $4^{\circ} \mathrm{C}$. The supernatants were transferred to a new tube, and protein concentrations were measured using a BCA Protein Assay Kit described above. To separate the mitochondrial and cytosolic fractions, a Mitochondria/Cytosol Fractionation Kit (Beyotime Inst. Biotech) was used according to the manufacturer's instructions. For western blots, equal amounts of protein $(40 \mu \mathrm{g})$ were resolved with SDS-PAGE and transferred to PVDF membranes (Bio-Rad, Hercules, CA, USA). The immunoblot membranes were incubated with blocking solution consisting of $5 \%$ milk in TBST for $2 \mathrm{~h}$ at room temperature and then incubated at $4{ }^{\circ} \mathrm{C}$ on a shaker overnight with the primary antibodies. The next day, the primary antibodies were removed, and the membranes were incubated with 1:2000 horseradish peroxidase-conjugated secondary antibodies (Cell Signaling Technology, Beverly, MA, USA) at $37^{\circ} \mathrm{C}$ for $1-2 \mathrm{~h}$. Antibody-binding bands were visualized with enzyme-catalyzed chemiluminescence (ECL, Beyotime Inst. Biotech). The density of the bands were quantified with Image Pro Plus version 6.0 software (Media Cybernetics, Bethesda, MD, USA) and normalized against a loading control. The following primary antibodies (Cell Signaling Technology or Santa Cruz Biotechnology, Santa Cruz, CA, USA) and dilutions were used: Insulin (sc-9168, 1:500), Bax (\#2772, $1: 2000)$, Bcl-2 (\#2876, 1:2000), Apaf-1 (\#8723S, 1:1000), caspase-9 (\#9506, $1: 1000)$, caspase-3 (\#9662, 1:1000), cytochrome $c(\# 4272,1: 1000), \beta$-actin (\#4976, 1:2000), GAPDH (\#2118:1:2000) and VDAC (\#4866:1:2000).

Statistical analyses. All data are expressed as the mean \pm S.E.M. The differences between the untreated control and BPA-exposed groups were analyzed by one-way analysis of variance (ANOVA) followed by Dunnett's T3 test. Differences with $\left.P<0.05\left(^{*}\right), P<0.01^{(* *}\right)$, or $\left.P<0.001^{(* *}\right)$ considered statistically significant. Analyses were conducted with SPSS (Statistical Package for the Social Sciences) software v.17.0 (SPSS Inc., Chicago, IL, USA).

\section{Conflict of Interest}

The authors declare no conflict of interest.
Acknowledgements. This work was supported by the 100 Talents Program of Chinese Academy of Sciences (KZCX2-YW-BR-18) and the National Natural Science Foundation of China $(21277137,21207128)$. We thank Hongyuan Luo for helping to perform Laser Scanning Confocal Microscope inspections.

1. Vandenberg LN, Maffini MV, Sonnenschein C, Rubin BS, Soto AM. Bisphenol-A and the great divide: a review of controversies in the field of endocrine disruption. Endocr Rev 2009; 30: 75-95.

2. Calafat AM, Ye X, Wong LY, Reidy JA, Needham LL. Exposure of the US population to bisphenol A and 4-tertiary-octylphenol: 2003-2004. Environ Health Perspect 2008; 116: 39.

3. Vandenberg LN, Hauser R, Marcus M, Olea N, Welshons WV. Human exposure to bisphenol A (BPA). Reprod Toxicol 2007; 24: 139-177.

4. Lang IA, Galloway TS, Scarlett A, Henley WE, Depledge M, Wallace RB et al. Association of urinary bisphenol A concentration with medical disorders and laboratory abnormalities in adults. JAMA 2008; 300: 1303-1310.

5. Wang T, Li M, Chen B, Xu M, Xu Y, Huang Y et al. Urinary bisphenol A (BPA) concentration associates with obesity and insulin resistance. J Clin Endocrinol Metab 2012; 97: E223-E227.

6. Melzer D, Rice NE, Lewis C, Henley WE, Galloway TS. Association of urinary bisphenol A concentration with heart disease: evidence from NHANES 2003/06. PLOS One 2010; 5: e8673.

7. Silver MK, O'Neill MS, Sowers MFR, Park SK. Urinary bisphenol a and type-2 diabetes in US adults: data from NHANES 2003-2008. PLoS One 2011; 6: e26868.

8. Shankar A, Teppala S. Relationship between urinary bisphenol A levels and diabetes mellitus. J Clin Endocrinol Metab 2011; 96: 3822-3826.

9. Ning G, Bi Y, Wang T, Xu M, Xu Y, Huang Y et al. Relationship of urinary bisphenol $A$ concentration to risk for prevalent type 2 diabetes in Chinese adults. Ann Intern Med 2011; 155: 368-374.

10. Batista TM, Alonso-Magdalena $\mathrm{P}$, Vieira E, Amaral MEC, Cederroth CR, Nef $S$ et al. Short-term treatment with bisphenol-A leads to metabolic abnormalities in adult male mice. PLoS One 2012; 7: e33814.

11. Nadal A, Ropero AB, Laribi O, Maillet M, Fuentes E, Soria B. Nongenomic actions of estrogens and xenoestrogens by binding at a plasma membrane receptor unrelated to estrogen receptor $\alpha$ and estrogen receptor $\beta$. Pro Natl Acad Sci 2000; 97: 11603-11608.

12. Quesada I, Fuentes E, Viso-León MC, Soria B, Ripoll C, Nadal A. Low doses of the endocrine disruptor bisphenol-A and the native hormone 17ß-estradiol rapidly activate transcription factor CREB. FASEB J 2002; 16: 1671-1673.

13. Alonso-Magdalena P, Ropero AB, Carrera MP, Cederroth CR, Baquie M, Gauthier BR et al. Pancreatic insulin content regulation by the estrogen receptor ER $\alpha$. PLoS One 2008; 3: e2069.

14. Soriano S, Alonso-Magdalena P, García-Arévalo M, Novials A, Muhammed SJ, Salehi A et al. Rapid Insulinotropic action of low doses of bisphenol-A on mouse and human islets of langerhans: role of estrogen receptor $\beta$. PLoS One 2012; 7: e31109.

15. Lowell BB, Shulman GI. Mitochondrial dysfunction and type 2 diabetes. Science 2005; $\mathbf{3 0 7}$ : 384-387.

16. Szendroedi J, Phielix E, Roden M. The role of mitochondria in insulin resistance and type 2 diabetes mellitus. Nat Rev Endocrinol 2011; 8: 92-103.

17. Maechler $P$, De Andrade P. Mitochondrial damages and the regulation of insulin secretion. Biochem Soc Trans 2006; 34: 824-827.

18. Wei J, Lin Y, Li Y, Ying C, Chen J, Song $L$ et al. Perinatal exposure to bisphenol $A$ at reference dose predisposes offspring to metabolic syndrome in adult rats on a high-fat diet. Endocrinology 2011; 152: 3049-3061. 
19. Song L, Xia W, Zhou Z, Li Y, Lin Y, Wei J et al. Low-level phenolic estrogen pollutants impair islets morphology and $\beta$-cells function in isolated rat islets. J Endocrinol 2012; 215: 303-311.

20. Skelin M, Rupnik M, Cencic A. Pancreatic beta cell lines and their applications in diabetes mellitus research. Altex 2010; 27: 105-113.

21. Vandenberg LN, Chahoud I, Heindel JJ, Padmanabhan V, Paumgartten FJR, Schoenfelder G. Urinary, circulating, and tissue biomonitoring studies indicate widespread exposure to bisphenol A. Environ Health Perspect 2010; 118: 1055-1070.

22. Makaji E, Raha S, Wade MG, Holloway AC. Effect of environmental contaminants on beta cell function. Int J Toxicol 2011; 30: 410-418.

23. Wang F, Hua J, Chen M, Xia Y, Zhang Q, Zhao R et al. High urinary bisphenol A concentrations in workers and possible laboratory abnormalities. Occup Environ Med 2012; 69: 679-684.

24. Wetherill YB, Akingbemi BT, Kanno J, McLachlan JA, Nadal A, Sonnenschein $C$ et al. In vitro molecular mechanisms of bisphenol A action. Reprod Toxicol 2007; 24: 178-198.

25. Welshons WV, Nagel SC, vom Saal FS. Large effects from small exposures. III. Endocrine mechanisms mediating effects of bisphenol A at levels of human exposure. Endocrinology 2006; 147: s56-s69.

26. Henquin JC. Regulation of insulin secretion: a matter of phase control and amplitude modulation. Diabetologia 2009; 52: 739-751.

27. Rorsman $\mathrm{P}$, Renström E. Insulin granule dynamics in pancreatic beta cells. Diabetologia 2003; 46: 1029-1045.

28. MacDonald PE, Joseph JW, Rorsman P. Glucose-sensing mechanisms in pancreatic $\beta$-cells. Philos Trans R Soc B Bio Sci 2005; 360: 2211-2225.

29. Polonsky KS, Semenkovich CF. The pancreatic $\beta$ cell heats up: UCP2 and insulin secretion in diabetes. Cell 2001; 105: 705-707.

30. Zhang CY, Baffy G, Perret P, Krauss S, Peroni O, Grujic D et al. Uncoupling protein-2 negatively regulates insulin secretion and is a major link between obesity, [beta] cell dysfunction, and type 2 diabetes. Cell 2001; 105: 745-755.

31. Chan CB, De Leo D, Joseph JW, McQuaid TS, Ha XF, Xu F et al. Increased uncoupling protein-2 levels in $\beta$-cells are associated with impaired glucose-stimulated insulin secretion. Diabetes 2001; 50: 1302-1310.
32. Hock MB, Kralli A. Transcriptional control of mitochondrial biogenesis and function. Annu Rev Physiol 2009; 71: 177-203.

33. vom Saal FS, Akingbemi BT, Belcher SM, Birnbaum LS, Crain DA, Eriksen M et al. Chapel Hill bisphenol A expert panel consensus statement: integration of mechanisms, effects in animals and potential to impact human health at current levels of exposure. Reprod toxicol 2007; 24: 131-138.

34. Wang Q, Zhao XF, Ji YL, Wang H, Liu P, Zhang C et al. Mitochondrial signaling pathway is also involved in bisphenol A induced germ cell apoptosis in testes. Toxicol Lett 2010; 199: $129-135$.

35. Xu J, Osuga Y, Yano T, Morita Y, Tang X, Fujiwara T et al. Bisphenol A induces apoptosis and G2-to-M arrest of ovarian granulosa cells. Biochem Biophys Res Commun 2002; 292: $456-462$.

36. Kroemer G, Reed JC. Mitochondrial control of cell death. Nat Med 2000; 6: 513-519.

37. Youle RJ, Strasser A. The BCL-2 protein family: opposing activities that mediate cell death. Nat Rev Molr Cell Biol 2008; 9: 47-59.

38. Earnshaw WC, Martins LM, Kaufmann SH. Mammalian caspases: structure activation, substrates, and functions during apoptosis. Annu Rev Biochem 1999; 68: 383-424.

39. Lakhani SA, Masud A, Kuida K, Porter Jr GA, Booth CJ, Mehal WZ et al. Caspases 3 and 7: key mediators of mitochondrial events of apoptosis. Science 2006; 311: 847-851.

40. Zimmermann KC, Bonzon C, Green DR. The machinery of programmed cell death. Pharmacol Ther 2001; 92: 57-70.

(c) Cell Death and Disease is an open-access journal published by Nature Publishing Group. This work is licensed under the Creative Commons Attribution-NonCommercialShare Alike 3.0 Unported License. To view a copy of this license, visit http://creativecommons.org/licenses/by-nc-sa/3.0/ 